DRAFT VERSION JULY 22, 2019

Preprint typeset using LTEX style emulateapj v. 12/16/11

\title{
A SEARCH FOR GAMMA-RAY PROMPT EMISSION ASSOCIATED WITH THE LORIMER BURST FRB 010724
}

\author{
C. Guidorzi ${ }^{1}$, M. Marongiu ${ }^{1,2}$, R. Martone ${ }^{1,2}$, L. Amati ${ }^{3}$, F. Frontera $^{1,3}$, L. NiCAstro $^{3}$, M. Orlandini $^{3}$, R. Margutti $^{4}$, \\ E. VIRGILLi ${ }^{1}$ \\ Draft version July 22, 2019
}

\begin{abstract}
No transient electromagnetic emission has yet been found in association to fast radio bursts (FRBs), the only possible exception ( $3 \sigma$ confidence) being the putative $\gamma$-ray signal detected in Swift/BAT data in the energy band $15-150 \mathrm{keV}$ at the time and position of FRB 131104. Systematic searches for hard X/ $\gamma-$ ray counterparts to other FRBs ended up with just lower limits on the radio/ $\gamma$-ray fluence ratios. In 2001, at the time of the earliest discovered FRBs, the BeppoSAX Gamma-Ray Burst Monitor (GRBM) was one of the most sensitive open sky $\gamma$-ray monitors in the 40-700 keV energy band. During its lifetime, one of the FRBs with the highest radio fluence ever recorded, FRB 010724 (800 $\pm 400 \mathrm{Jy} \mathrm{ms})$, also known as the "Lorimer burst", was promptly visible to the GRBM. Upon an accurate modeling of the GRBM background, eased by its equatorial orbit, we searched for a possible $\gamma$-ray signal in the first $400 \mathrm{~s}$ following the FRB, similar to that claimed for FRB 131104 and found no significant emission down to a $5 \sigma$ limit in the range $(0.24-4.7) \times 10^{-6} \mathrm{erg} \mathrm{cm}^{-2}$ (corresponding to 1 and $400 \mathrm{~s}$ integration time, respectively), in the energy band $40-700 \mathrm{keV}$. This corresponds to $\eta=F_{\text {radio }} / F_{\gamma}>10^{8-9} \mathrm{Jy} \mathrm{ms} \mathrm{erg}^{-1} \mathrm{~cm}^{2}$, i.e. the deepest limit on the ratio between radio and $\gamma-$ ray fluence, which rules out a $\gamma$-ray counterpart similar to that of FRB 131104. We discuss the implications on the possible mechanisms and progenitors that have been proposed in the literature, also taking into account its relatively low dispersion measure $\left(375 \pm 3 \mathrm{pc} \mathrm{cm}^{-3}\right)$ and an inferred redshift limit of $z<0.4$.
\end{abstract}

Subject headings: stars: individual (FRB 010724) - radiation mechanisms: non-thermal

\section{INTRODUCTION}

Fast radio bursts (FRBs) are ms-long bright pulses of unknown origin characterized by a dispersion measure (DM) significantly in excess of the corresponding Galactic value (Lorimer et al. 2007; Thornton et al. 2013). A large DM value, due to a relatively large electron column density integrated along the sightline, strongly hints at an extragalactic origin. Despite the high all-sky daily rate of several thousands (e.g., Rane et al. 2016; Crawford et al. 2016; Scholz et al. 2016), only $\sim 80$ FRBs have been publicly announced so far (as of July 2019; Petroff et al. 2016). This is mainly due to the relatively narrow fields of view (FOV) of most of the radiotelescopes that first observed them, although the discovery rate is now ramping up thanks to a new generation of wide FOV experiments that have recently come on stage. The majority of them appear to be one-off events, except for a couple of them (Spitler et al. 2016; CHIME/FRB Collaboration et al. 2019). See Petroff et al. (2019a); Katz (2018); Popov et al. (2018); Rane \& Lorimer (2017) for updated reviews.

Affected by several arcmin positional uncertainty, FRBs so far defied any search for either prompt simultaneous or afterglow transient emission at other wavelengths that would help identify their host galaxies, measure the distance, constrain the radiation mechanism and the nature of the progenitor. There are a few exceptions: FRB 121102, whose host was found at a redshift of $z=0.193$ (Tendulkar et al. 2017), which is also one of the two repeating sources: it was precisely

\footnotetext{
${ }^{1}$ Department of Physics and Earth Science, University of Ferrara, via Saragat 1, I-44122, Ferrara, Italy

2 ICRANet, Piazza della Repubblica 10, I-65122 Pescara, Italy

${ }^{3}$ INAF-Osservatorio di Astrofisica e Scienza dello Spazio di Bologna, Via Piero Gobetti 93/3, I-40129 Bologna, Italy

${ }^{4}$ Center for Interdisciplinary Exploration and Research in Astrophysics and Department of Physics and Astronomy, Northwestern University, 2145 Sheridan Road, Evanston, IL 60208-3112, USA
}

its repeating nature that made it possible to localize with sub-arcsec accuracy during interferometric radio observations (Chatterjee et al. 2017). Recently, the redshifts of two other non-repeating FRBs have been determined: FRB 180924 at $z=0.3214$ (Bannister et al. 2019) and FRB 190523 at $z=0.66$ (Ravi et al. 2019).

A possible $(3 \sigma$ confidence $) \gamma-$ ray $\sim 400$-s long transient source, Swift J0644.5-5111, positionally and temporally compatible with FRB 131104 was reported by DeLaunay et al. (2016, hereafter D16) in the $15-150 \mathrm{keV}$ band within the data of the Burst Alert Telescope (BAT; Barthelmy et al. 2005) aboard the Neil Gehrels Swift Observatory (Gehrels et al. 2004). The possibility of a gamma-ray burst (GRB) associated to this FRB was later called into question by the lack of any radio afterglow (Shannon \& Ravi 2017). However, admitting microphysical shock parameter values different from what is typically assumed for GRB afterglows, this GRB-FRB association remains plausible (Gao \& Zhang 2017). A similar search carried out by D16 for other FRBs that were promptly visible with BAT yielded only upper limits. Previous searches for high-energy counterparts to FRBs had ended up with lower limits to the radio/ $\gamma$ fluence ratio $\eta_{\text {frb }}>10^{7-9} \mathrm{Jy} \mathrm{ms} \mathrm{erg}^{-1} \mathrm{~cm}^{2}$, incompatible with the upper limit $\eta_{\text {sgr }}<10^{7} \mathrm{Jy} \mathrm{ms} \mathrm{erg}^{-1} \mathrm{~cm}^{2}$ derived for the $\gamma$-ray giant flare of the Galactic magnetar SGR 1806-20 (Tendulkar et al. 2016). Similarly, Scholz et al. (2017) analyzed the data of the Gamma-ray Burst Monitor (Meegan et al. 2009) aboard the Fermi Gamma-Ray Telescope and found nothing down to $10^{-8} \mathrm{erg} \mathrm{cm}^{-2}$ in the 10 $100 \mathrm{keV}$ energy band in a 2-s window centered on the arrival times of four bursts of repeater FRB 121102. Reversing the approach, Palaniswamy et al. (2014) found no prompt radio pulses compatible with FRBs associated to five GRBs that were observed starting within $140 \mathrm{~s}$ of the $\gamma-$ ray trigger time. No detections were reported from searches 
in the GeV domain using the Fermi Large Area Telescope (Atwood et al. 2009) for both one-off FRBs (Yamasaki et al. 2016; Xi et al. 2017) and repeaters (Zhang \& Zhang 2017; Yang et al. 2019). Analogous results were obtained at very high energies, above $100 \mathrm{GeV}$ (H.E.S.S. Collaboration et al. 2017; MAGIC Collaboration et al. 2018) as well as for TeV$\mathrm{PeV}$ neutrinos coincident with FRBs Aartsen et al. 2018; Albert et al. 2019).

One of the open questions is whether a common family of progenitors is responsible for the observed population of FRBs, in particular one-off and repeating sources (Palaniswamy et al.2018; Caleb et al.2019), or, as in the case of GRBs, at least two families are required to explain the long and short duration events respectively associated to the core collapse of some kind of hydrogen-stripped massive stars and to the merging of compact objects (see Kumar \& Zhang 2015 for a review). Thus, the existence of repeating sources does not necessarily rule out the so-called cataclysmic models. A number of the proposed models that predict an associated high-energy emission invoke rapidly rotating young neutron stars (NS) and magnetars as progenitors (Katz 2014; Cordes \& Wasserman 2016; Lyutikov et al.2016). Given also that in some models ms magnetars are supposed to form following the core-collapse that powers both long (Usov 1992; Thompson 1994; Bucciantini et al. 2007; Metzger et al. 2011) and short GRBs (Fan \& Xu|2006; Metzger et al.|2008; Rowlinson et al. 2010; Gompertz et al. 2013), as well as superluminous SNe (SLSNe; Nicholl et al. 2017; Metzger et al. 2017), it is worth searching for hard $\mathrm{X} / \gamma-$ ray emission associated to FRBs. As with GRBs and SNe the study of the environments may potentially provide useful clues on the homogeneity of the FRB progenitors (Eftekhari \& Berger 2017).

Motivated by the results of D16, we exploited the sensitivity of the Gamma-Ray Burst Monitor (GRBM; Frontera et al. 1997) that operated as an open-sky detector in the energy band 40-700 keV aboard BeppoSAX (Boella et al. 1997) in 1996-2002. We carried out a detailed search for a highenergy counterpart to FRB 010724, the first reported FRB (Lorimer et al. 2007), popularly known as the Lorimer burst: this was so bright that it saturated the Parkes multibeam receiver and was also detected in three other beams of the receiver. From an initial estimate of $150 \mathrm{Jy} \mathrm{ms}$, its fluence has recently been revised to $800 \pm 400 \mathrm{Jy}$ ms (Ravi 2019). Owing to its exceptional brightness, FRB 010724 might represent a discovery bias (Macquart \& Ekers 2018) and may come from a space-limited population, whose local density would far exceed the cosmological one (Katz 2016). Given its relatively low DM of $375 \pm 3 \mathrm{pc} \mathrm{cm}^{-3}$, evidence that this FRB might be relatively nearby is also provided by the brightness-dispersion correlation shown in the ASKAP sample (Shannon et al. 2018; see also Niino 2018). In this respect, another example of low-DM/high-flux FRB is given by FRB 110214 (Petroff et al. 2019b). We therefore discuss the implications of our results on the proposed associations as a function of the possible redshift compatibly with its DM. Hereafter, we assume the latest Planck cosmological parameters: $H_{0}=67.74 \mathrm{~km} \mathrm{~s}^{-1} \mathrm{Mpc}^{-1}, \Omega_{m}=0.31, \Omega_{\Lambda}=0.69$ (Planck Collaboration et al. 2016).

\section{DATA ANALYSIS}

From the FRB catalog (Petroff et al. 2016) we selected the four FRBs occurred in 2001 during the BeppoSAX operational

\footnotetext{
5 http://www.frbcat.org
}

life (Table 1) and checked the availability of GRBM data at the time of each FRB along with its visibility (i.e., not Earthblocked). Only FRB 010724 passed the selection. Based on the direction of FRB 010724 with reference to the BeppoSAX frame, the expected signal for each of the four GRBM units is comparable with one another (Table 2). From the BeppoSAX GRB catalog (Frontera et al. 2009) we used the response matrices of the GRB with the nearest local position $\left(158^{\circ},-46^{\circ}\right)$ to that of the FRB $\left(162^{\circ},-43.5^{\circ}\right)$, and for each unit we calculated the expected number of counts for both energy bands 40-700 $\mathrm{keV}$ and $>100 \mathrm{keV}$, assuming the same spectral models and $\gamma$-ray fluences found by D16 for FRB 131104 (Table 2). No prompt BeppoSAX X-ray data are available for FRB 010724, since it lies outside the FOV of the Wide Field Cameras (Jager et al. 1997).

We modeled the background rates for each unit and energy band by exploiting the GRBM data sharing the most similar configuration and that were acquired about one day before the FRB. A detailed description is reported in Appendix A

\section{RESULTS}

In order to investigate a potential $\gamma$-ray counterpart, we assumed a fast-rise slow-decay pulse profile as modeled by Kocevski et al. (2003) so as to have the desired duration of $\sim 400 \mathrm{~s}$. We chose the following parameters: rise and decay indices $r=2$ and $d=3$, respectively; peak time $t_{m}=100 \mathrm{~s}$ and $t_{0}=0$. Normalizations were chosen so as to match the expected counts (Sect. 2) in the time interval from 0 to $400 \mathrm{~s}$, comparable with the $T_{90}=377$ s of the Swift event. The resulting profiles are shown in Figure 1 with dashed lines, added to the background level, for the $40-700 \mathrm{keV}$ channels of each of the detectors, as well as for the average of the four, which has the best signal-to-noise ratio $(\mathrm{S} / \mathrm{N})$. Specifically, blue and orange lines correspond to the best-fit power-law (hereafter POW) and thermal bremsstrahlung (hereafter TB) models, respectively. Furthermore, we integrated the backgroundsubtracted counts in the $0-400 \mathrm{~s}$ time intervals and found no significant $(>5 \sigma)$ excess. The most constraining upper limit to the $40-700 \mathrm{keV}$ fluence is obtained from the mean time profile: a total count fluence of $247 \pm 273$ turns into a $5 \sigma$ upper limit of $F_{\gamma}(40-700)<4.7 \times 10^{-6} \mathrm{erg} \mathrm{cm}^{-2}$ for the POW and $F_{\gamma}(40-700)<7.1 \times 10^{-6} \mathrm{erg} \mathrm{cm}^{-2}$ for the TB model, accounting for the uncertainties on the best fit models. Assuming shorter $\gamma$-ray events and thus, integrating over shorter time intervals $\Delta t$, we found no $>5 \sigma$ excess, with the corresponding upper limits scaling approximately as $\sqrt{\Delta t}$. In particular, taking $\Delta t=1 \mathrm{~s}$, i.e. the time bin of the FRB, limits scale down by a factor of $\sim 19: F_{\gamma}(40-700)<2.5 \times 10^{-7} \mathrm{erg} \mathrm{cm}^{-2}$ and $<3.7 \times 10^{-7} \mathrm{erg} \mathrm{cm}^{-2}$ for the POW and TB models, respectively. Figure 2 shows the cumulative count fluence and corresponding values in physical units for a power-law spectrum (using $\Gamma=1.84$, which gives the most conservative limit on fluence) as a function of the integration time, along with the $5 \sigma$ upper limit. The most significant excess in $40-700 \mathrm{keV}$ is $3.3 \sigma$ around $t=14 \mathrm{~s}$. However, inspecting the individual detector profiles from Figure 1 suggests that it is mostly due to unit 3 and due to the background modeling inaccuracy rather than a genuine signal.

Consequently, a $\gamma$-ray counterpart equal to that of FRB 131104 cannot be ruled out for the TB model, whereas the POW model is rejected. Moreover, assuming a similar radio $-\gamma$ spectral slope as that found by D16, such a $\gamma$-ray counterpart is rejected with high confidence in the case of 
TABLE 1

BeppoSAX/GRBM DATA AVAILABILITY FOR THE FRBS DETECTED IN 2001.

\begin{tabular}{|c|c|c|c|c|c|c|c|c|}
\hline FRB & UT & $\begin{array}{c}\mathrm{RA}(\mathrm{J} 2000) \\
\left({ }^{\circ}\right)\end{array}$ & $\begin{array}{c}\mathrm{DEC}(\mathrm{J} 2000) \\
\left({ }^{\circ}\right)\end{array}$ & $\begin{array}{c}\phi \\
\left({ }^{\circ}\right)\end{array}$ & $\begin{array}{c}\theta \\
\left(^{\circ}\right)\end{array}$ & $\begin{array}{c}\text { Elevation } \\
\left({ }^{\circ}\right)\end{array}$ & $\mathrm{OP}$ & Comment \\
\hline 010125 & $00: 29: 14$ & 286.7 & -40.7 & 42.2 & 19.4 & -18.0 & 10600 & Earth blocked \\
\hline $010312^{\mathrm{a}}$ & $11: 06: 48$ & 81.7 & -64.9 & - & - & 1.4 & 10862 & no data \\
\hline 010621 & 13:02:09 & 283.0 & -8.5 & 203.5 & -8.8 & 59.5 & 11411 & unavailable due to data gap \\
\hline 010724 & 19:50:00 & 19.5 & -75.2 & 162.0 & -43.5 & 38.6 & 11651 & visible and available \\
\hline
\end{tabular}

Note. $-(\phi, \theta)$ is the direction with respect to the BeppoSAX payload reference frame (Frontera et al. 2009). The elevation angle is calculated with respect to the Earth limb. OP is the observing period, which identifies the data set in the BeppoSAX archive.

${ }^{a}$ Reported by Zhang et al. (2019).

TABLE 2

EXPECTED NET COUNTS FOR A $\gamma-$ RAY EVENT EQUAL TO THAT FOUND FOR FRB $131104^{\mathrm{a}}$.

\begin{tabular}{|c|c|c|c|c|c|c|c|c|c|c|}
\hline Model $^{\mathrm{b}}$ & $\Gamma$ & $\begin{array}{c}k T \\
(\mathrm{keV})\end{array}$ & $\begin{array}{c}F_{\gamma}(40-700) \\
\left(\mathrm{erg} \mathrm{cm}^{-2}\right)\end{array}$ & $\begin{array}{l}\text { Band } \\
(\mathrm{keV})\end{array}$ & $\begin{array}{l}\text { GRBM1 } \\
\text { (counts) }\end{array}$ & $\begin{array}{l}\text { GRBM2 } \\
\text { (counts) }\end{array}$ & $\begin{array}{l}\text { GRBM3 } \\
\text { (counts) }\end{array}$ & $\begin{array}{l}\text { GRBM4 } \\
\text { (counts) }\end{array}$ & $\begin{array}{c}\text { Mean } \\
\text { (counts) }\end{array}$ & $\begin{array}{c}5 \sigma \text { U.L. on } F_{\gamma}{ }^{\mathrm{c}} \\
\quad\left(\mathrm{erg} \mathrm{cm}^{-2}\right)\end{array}$ \\
\hline POW & 1.16 & - & 5 & & & & & & $\begin{array}{l}5109 \\
4707\end{array}$ & $<4.0 \times 10^{-6}$ \\
\hline POW & $0.38^{\mathrm{d}}$ & - & $4.9 \times 10^{-5}$ & $\begin{array}{r}40-700 \\
>100\end{array}$ & $\begin{array}{l}16754 \\
16303\end{array}$ & $\begin{array}{l}11971 \\
11370\end{array}$ & $\begin{array}{l}17188 \\
16722\end{array}$ & $\begin{array}{l}25820 \\
26938\end{array}$ & $\begin{array}{l}17933 \\
17833\end{array}$ & $<3.7 \times 10^{-6}$ \\
\hline POW & $1.84^{\mathrm{d}}$ & $\begin{array}{l}- \\
-\end{array}$ & $6.1 \times 10^{-6}$ & $\begin{array}{r}40-700 \\
>100\end{array}$ & & $\begin{array}{l}1316 \\
1024\end{array}$ & $\begin{array}{l}1696 \\
1338\end{array}$ & & $\begin{array}{l}1752 \\
1447\end{array}$ & $<4.7 \times 10^{-6}$ \\
\hline POW & 2 & $\begin{array}{l}- \\
-\end{array}$ & $5.0 \times 10^{-6}$ & $\begin{array}{r}40-700 \\
>100\end{array}$ & $\begin{array}{r}1259 \\
991\end{array}$ & $\begin{array}{r}1055 \\
793\end{array}$ & $\begin{array}{l}1332 \\
1012\end{array}$ & $\begin{array}{l}1852 \\
1600\end{array}$ & $\begin{array}{l}1374 \\
1099\end{array}$ & $<5.0 \times 10^{-6}$ \\
\hline $\mathrm{TB}$ & - & 200 & $4.2 \times 10^{-6}$ & $\begin{array}{r}40-700 \\
>100\end{array}$ & $\begin{array}{r}1017 \\
741\end{array}$ & $\begin{array}{l}896 \\
628\end{array}$ & $\begin{array}{r}1097 \\
760\end{array}$ & $\begin{array}{l}1472 \\
1182\end{array}$ & $\begin{array}{r}1120 \\
828\end{array}$ & $<4.7 \times 10^{-6}$ \\
\hline $\mathrm{TB}$ & - & 75 & $2.3 \times 10^{-6}$ & $\begin{array}{r}40-700 \\
>100\end{array}$ & $\begin{array}{l}410 \\
227\end{array}$ & $\begin{array}{l}405 \\
218\end{array}$ & $\begin{array}{l}439 \\
219\end{array}$ & $\begin{array}{l}522 \\
325\end{array}$ & $\begin{array}{l}444 \\
247\end{array}$ & $<7.1 \times 10^{-6}$ \\
\hline
\end{tabular}

a Counts are meant to be in excess of the background for each GRBM unit for a $\gamma$-ray event with $F_{\gamma}(15-150 \mathrm{keV})=$ $4 \times 10^{-6} \mathrm{erg} \mathrm{cm}^{-2}$.

${ }^{\mathrm{b}}$ The spectral models are the same as in D16: power-law (POW) and thermal bremsstrahlung (TB).

${ }^{c}$ Upper limits obtained by integrating the background-subtracted mean counts over the $[0,400] \mathrm{s}$ interval.

${ }^{\mathrm{d}}$ Photon index values corresponding to the confidence interval estimated by D16.

FRB 010724, given that the Lorimer burst has a radio fluence $340 \pm 170$ times as high as that of FRB 131104 .

This $\gamma$-ray upper limit is the most constraining value for the radio-to- $\gamma$ fluence ratio yet obtained for an FRB: $\log _{10} \eta>8.2$ for POW and $\log _{10} \eta>8.0$ for the TB model. These values are $\sim 250$ times higher than the value measured for FRB 131104, $\log _{10} \eta=5.8 \pm 0.2$ (D16). Taking the limit derived for the 1 -s bin centered on the FRB, the same limits on $\eta$ become $\log _{10} \eta>9.5-9.3$ for the POW and TB models, respectively. For reference, we also considered the case of a power-law with $\Gamma=2$ (Table 2): the most conservative upper limit changes only marginally.

As a further check, we exploited the 240-channel spectra continuously acquired by the GRBM units in the $40-700 \mathrm{keV}$ energy band every $128 \mathrm{~s}$ (Frontera et al. 1997; Guidorzi et al. 2011) to investigate the possible presence of a signal in two energy bands: $40-100$ and $40-200 \mathrm{keV}$, respectively. Figure 3 displays the corresponding 128-s light curves averaged over the four units (black) along with the analogous data of the background orbit (red). Although the poorer temporal resolution does not allow us to model the background as accurately as we did for the 1-s ratemeters, yet the comparison between the FRB orbit and the background orbit rates excludes the possibility of a relatively soft signal associated with the FRB.

\section{DISCUSSION}

We rule out a $\gamma$-ray transient event that is similar to Swift J0644.5-5111 for FRB 131104 (cf. D16). This is how- ever assuming a power-law spectrum; the softer case of a thermal bremsstrahlung is only marginally excluded. A $\gamma$-ray signal, rescaled to the much higher radio fluence of the Lorimer burst, is also ruled out, regardless of the spectral mode. Therefore, if the association found by D16 is true, the Lorimer burst is intrinsically different and much more $\gamma$-ray quiet. Alternatively, the Lorimer burst was associated with an analogous $\gamma-$ ray transient that was more collimated than the radio emission and that was not pointing towards Earth (e.g., Romero et al. 2016).

For FRB 010724 the DM excess, i.e. the measured DM removed of the Galactic contribution, is $\mathrm{DM}_{\mathrm{E}}=330 \mathrm{pc} \mathrm{cm}^{-3}$ (Petroff et al. 2016) and can be expressed as the sum of different terms: the intergalactic medium (IGM), the host galaxy $(\mathrm{HG})$, and the local environment surrounding the FRB source:

$$
\mathrm{DM}_{\mathrm{E}}=\mathrm{DM}_{\mathrm{IGM}}+\frac{\mathrm{DM}_{\mathrm{HG}}+\mathrm{DM}_{\mathrm{loc}}}{1+z} .
$$

Using the relation by Zhang (2018) $z \sim D_{\mathrm{IGM}} /\left(855 \mathrm{pc} \mathrm{cm}^{-3}\right)$, an upper limit on redshift of $z<0.4$ is obtained. Simulations suggest that the contribution of the host to the observed DM ranges from an average value of $45 \mathrm{pc} \mathrm{cm}^{-3}$ in the case of a dwarf galaxy all the way up to $\sim 142 \mathrm{pc} \mathrm{cm}^{-3}$ averaging over all inclination angles for a spiral (Xu \& Han 2015).

Figure 4 summarizes our constraints for various models considered. Assuming that the host is a dwarf, similarly to long GRBs and SLSNe as is the case for the repeater FRB 121101 (Tendulkar et al.2017; Metzger et al.2017), and 


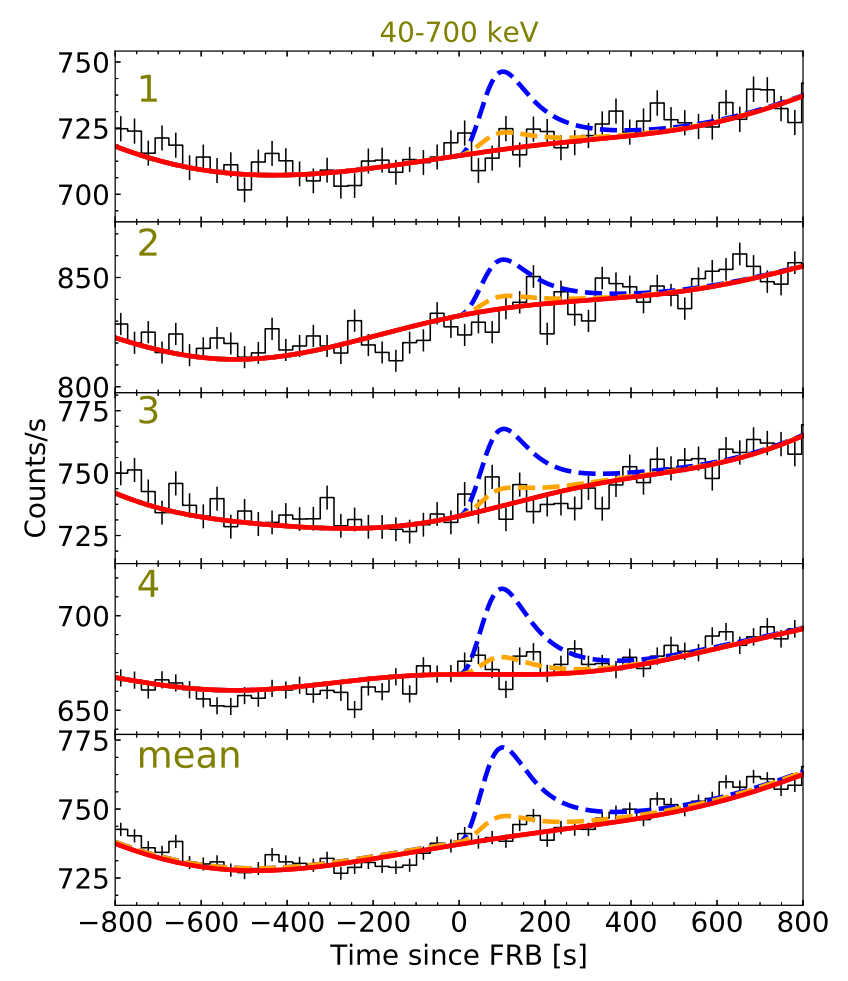

FIG. 1.-BeppoSAX/GRBM ratemeters (40-700 keV; bin time of $32 \mathrm{~s})$ at the time of FRB 010724. Background (solid lines) was obtained modeling a different orbit. From top to bottom: individual units 1 to 4 , the mean of the four units. Dashed blue (orange) profile is what one would expect for an event equal to that found by D16 in Swift/BAT data for FRB 131104 extrapolating the best-fit power-law (thermal bremsstrahlung) model to the $40-700 \mathrm{keV}$ band.

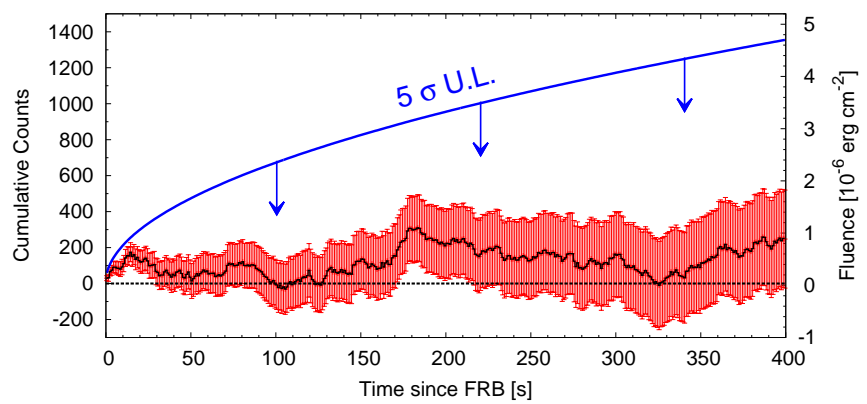

FIG. 2.- Cumulative net counts in the $40-700 \mathrm{keV}$ energy band averaged over the four GRBM units as a function of integration time, from 0 to $t$, with $t$ spanning the $0-400 \mathrm{~s}$ interval. Error bars are $1 \sigma$. The solid blue line shows the corresponding $5 \sigma$ upper limits. The corresponding fluence (right-hand axis) is calculated assuming a power-law spectrum with $\Gamma=1.84$.

adopting for $\mathrm{DM}_{\mathrm{HG}}$ the typical value expected for this kind of galaxies, the constraint on redshift becomes $z \lesssim 0.3$. Using the typical value for a spiral, i.e. about half of $\mathrm{DM}_{\mathrm{E}}$, the range shrinks to $z \lesssim 0.2$. The same constraint holds assuming that half of $\mathrm{DM}_{\mathrm{E}}$ is due to the local environment of the FRB progenitor. Making no assumption on $\mathrm{DM}_{\mathrm{E}}$, the energy of the radio pulse itself lies in the range $10^{38}<E_{\text {radio }} / \mathrm{erg}<6 \times 10^{42}$, in line with what has been argued for other FRBs, although at

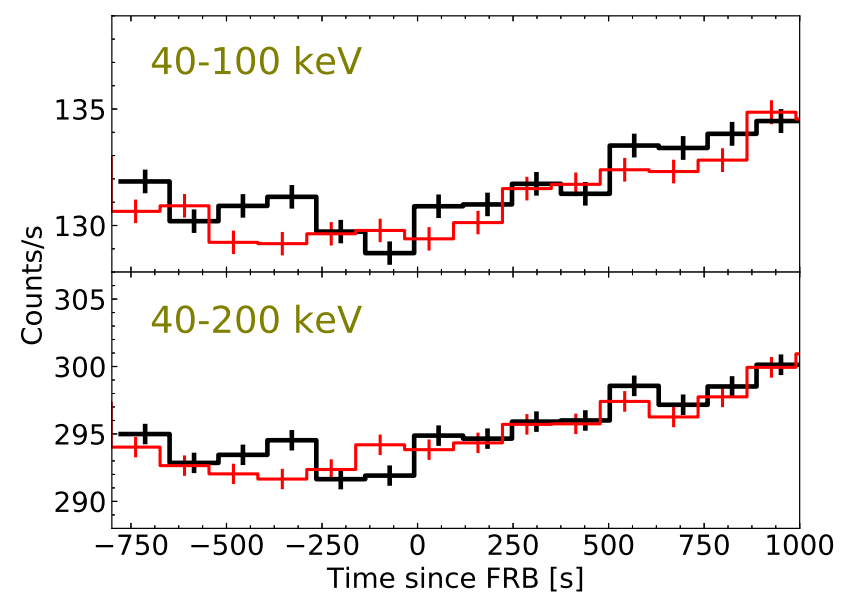

FIG. 3.- Mean count rates in two different energy bands with an integration time of $128 \mathrm{~s}$, obtained from the 240-channel spectra continuously acquired by the GRBM units every $128 \mathrm{~s}$. The FRB orbit rates (black) are shown together with the background orbit ones (red).

least 10 times more energetic than the individual bursts of the repeater FRB 121102 (Gourdji et al. 2019).

It is interesting to compare our limits on the $\gamma$-ray released energy with that of other astrophysical transients that have possible connections with FRBs. As shown in Figure 4, based on our analysis, we rule out the following interpretations:

- a $\gamma$-ray spike due to an extragalactic magnetar giant flare with the same radio/ $\gamma$-ray fluence ratio as that of the Galactic magnetar SGR 1806-20 (see also Tendulkar et al. 2016);

- short GRBs with isotropic-equivalent energies $E_{\gamma, \text { iso }}>$ $10^{50} \mathrm{erg}$, that are the majority of the observed population $\left(10^{49}-10^{52} \mathrm{erg}\right.$; Fong et al. 2015);

- the typical $\gamma$-ray emission of a cosmological long GRB, whose isotropic-equivalent energy ranges from $\sim 10^{51}$ up to $\sim 10^{55}$ erg (Tsvetkova et al. 2017).

On the other hand, we cannot reject the following scenarios:

- an extragalactic giant flare as energetic as the few ones observed in our Galaxy $\left(3 \times 10^{44}-2 \times 10^{46} \mathrm{erg}\right.$; Mazets et al. 1979; Feroci et al. 2001; Palmer et al. 2005; Hurley et al. 2005; orange region in Figure 4), regardless of the radio/ $\gamma$-ray spectral slope;

- a relatively weak short GRB ( $E_{\gamma, \text { iso }}$ in the range $10^{49}$ $\left.10^{50} \mathrm{erg}\right)$ at $z>0.04\left(D_{\mathrm{L}}>180 \mathrm{Mpc}\right)$

- a sub-energetic short GRB at $z>0.01\left(D_{\mathrm{L}}>45 \mathrm{Mpc}\right)$, such as GRB 170817A associated to GW 170817, the first binary neutron star (BNS) merger detected with gravitational interferometers (Abbott et al.2017a), which had $E_{\gamma, \text { iso }}=(5.3 \pm 1.0) \times 10^{46} \mathrm{erg}$. This holds true regardless of whether the GRB is truly subenergetic or is a typical short GRB viewed off-axis, as was the case of GRB 170817A (Margutti et al. 2017, 2018; Alexander et al. 2017, 2018; Troja et al. 2017; Granot et al. 2017); 


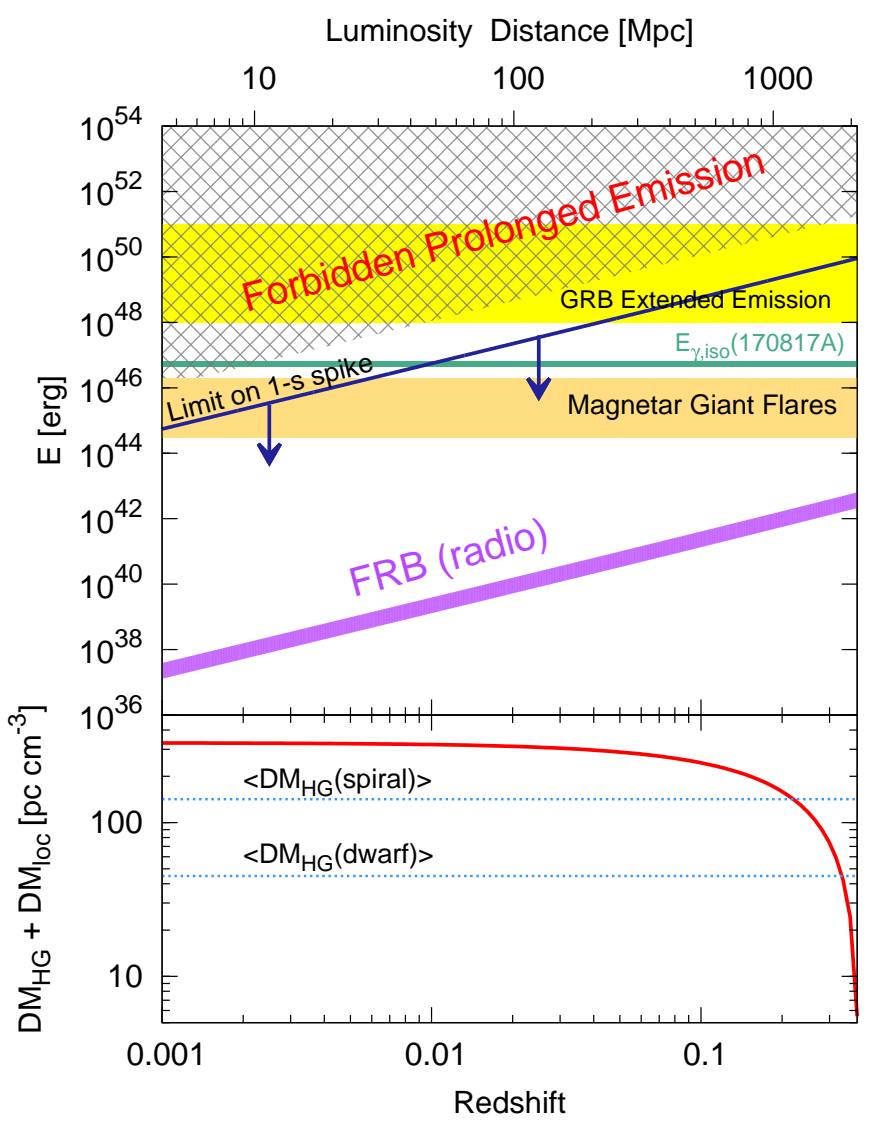

FIG. 4.- Top: emitted energy of the FRB (purple) and $5 \sigma$ upper limits on the 400-s prolonged emission (lower boundary of the hatched region) and on the 1-s coincident with the FRB (dark blue). For comparison, also shown are the isotropic-equivalent energies of the extended emission often associated to short GRBs (yellow), which is roughly as energetic as the short GRB itself, the sub-luminous short GRB 170817A associated to the double NS merger GW 170817 (green), magnetar giant flares (orange). Bottom: DM (red) due to the combination of the host galaxy (HG) and the local environment around the source (loc), estimated subtracting the IGM contribution from the excess $\mathrm{DM}$, as a function of redshift. The maximum value of $z=0.4$ corresponds to the case where all of the DM in excess of the Galactic value is due to the IGM. Also shown are the mean values of the expected DM due to the sightline within the host galaxy for two different kinds, dwarf and spiral galaxies, from simulations by Xu \& Han (2015).

- the presence of the so-called extended emission (EE) that characterizes some short GRBs. This is a faint, long-lasting $(\sim$ few hundred seconds $)$ hard $\mathrm{X} / \gamma$-ray tail following the initial spike (Lazzati et al. 2001; Montanari et al. 2005; Norris \& Bonnell 2006), whose isotropic-equivalent energy is in the range $10^{48}$ $10^{51}$ erg (Kisaka et al. 2017; yellow area in Fig. 44;

- the so-called low-luminosity (ll) long GRBs Kulkarni et al. 1998; Campana et al. 2006; Waxman et al. 2007) having $E_{\gamma \text {,iso }} \sim 10^{48}-10^{50} \mathrm{erg}$. $l l$-GRBs probably represent a separate family (Liang et al. 2007; Amati et al. 2007; Virgili et al. 2009; Wanderman \& Piran 2010; Howell \& Coward 2013) and are possibly the result of a relativistic shock breakout (Nakar \& Sari 2012).

Further considerations on the possible association between FRBs and GRBs and the other classes mentioned above are based on the relative volumetric rates. Although the FRB rate does depend on the redshift distribution, which is es- sentially unknown, yet using $\mathrm{DM}_{\mathrm{E}}$ as a proxy for distance and assuming that the observed population is uniformily distributed within $z \lesssim 1$, a rate of $\lesssim 10^{4} \mathrm{Gpc}^{-3} \mathrm{yr}^{-1}$ is obtained (Rane et al. 2016; Scholz et al. 2016; Crawford et al. 2016), which is not too different from the local one of $\mathrm{Ib} / \mathrm{c} \mathrm{SNe}$ (Dahlen et al. 2004; Li et al. 2011; Cappellaro et al. 2015). Our conjecture that long (i.e., not $l l$-) cosmological GRBs are not associated with the Lorimer burst is consistent with the different local volumetric rate of GRBs: $1.3_{-0.7}^{+0.6} f_{b}^{-1} \mathrm{Gpc}^{-3} \mathrm{yr}^{-1}$ Wanderman \& Piran 2010) is 10-100 times lower than that of FRBs even after accounting for a $f_{b}^{-1}=100$ beamingcorrected fraction. Somewhat higher values are obtained for short GRBs, whose rate is $4-8 f_{h}^{-1} \mathrm{Gpc}^{-3} \mathrm{yr}^{-1}$ (Coward et al. 2012; Wanderman \& Piran 2015). Although poorly constrained, the BNS rate of $1540_{-1220}^{+3200} \mathrm{Gpc}^{-3} \mathrm{yr}^{-1}$ from the recent gravitational wave observations (Abbott et al. 2017b) is not too far from that of FRBs. While a $l l$-GRB associated with the Lorimer burst is not excluded, their estimated local rate $\left(\sim 200-300 f_{h}^{-1} \mathrm{Gpc}^{-3} \mathrm{yr}^{-1}\right.$ with a lower $f_{h}^{-1}$ than for long GRBs; Soderberg et al. 2006; Liang et al. 2007) is also significantly lower than that of FRBs.

\section{CONCLUSIONS}

Motivated by the possible and controversial detection of a transient hard $\mathrm{X} / \gamma$-ray source positionally and temporally compatible with FRB 131104, we considered the brightest Lorimer FRB and examined the data of BeppoSAX/GRBM, one of the most sensitive instruments that in principle could have detected any simultaneous emission in the $\mathrm{keV}-\mathrm{MeV}$ band. A previous upper limit on the $\gamma$-ray fluence of $2 \times$ $10^{-7} \mathrm{erg} \mathrm{cm}^{-2}$ in the Konus/WIND passband was already adopted in the past (Tendulkar et al. 2016). However, this value is not the outcome of any specific data analysis, but merely corresponds to the lowest fluence detected in the Konus/WIND GRB catalog: this is because (i) the detection efficiency in the lowest end of the fluence distribution is likely to be significantly below 1; (ii) the typical duration of a GRB is one order of magnitude less than the transient signal possibly associated with FRB 131104; (iii) no specific analysis in that energy band at the time of the Lorimer burst has been reported yet. Based on our analysis we rule out a $\gamma$-ray counterpart for the Lorimer burst with the same radio/ $\gamma$-ray fluence ratio as that of FRB 131104. Furthermore, the combination of a very high specific flux and a relatively low DM strongly suggests this FRB to be a relatively nearby FRB. We used our fluence upper limits to investigate a possible relation of FRB sources with other classes of transient phenomena. We rule out an extragalactic magnetar flare analogous to that of the Galactic source SGR 1806-20, as well as a typical long GRB. Instead, a low-luminosity GRB or a sub-energetic short GRB (such as a standard short GRB viewed off-axis, as was probably the case of GRB 170817A associated to the first binary NS merger) cannot be excluded. These results, along with the comparison of the relative volumetric rates of the corresponding populations, do not rule out the possible association of FRBs with either binary NS mergers or with $l l$-GRBs, which, however, looks incompatible with a naive one-to-one correspondence.

We thank the anonymous referee for the detailed comments that improved the paper. We thank Marcello Giroletti for his careful reading and comments. Support for this work was provided by Università di Ferrara through grant FIR 2018 “A 
Broad-band study of Cosmic Gamma-Ray Burst Prompt and Afterglow Emission" (PI Guidorzi). This work is part of the
INAF PRIN SKA-CTA 2016. L.A. acknowledges financial contribution from the agreement ASI-INAF n.2017-14-H.O.

\section{REFERENCES}

Aartsen, M. G., Ackermann, M., Adams, J., et al. 2018, ApJ, 857, 117 Abbott, B. P., Abbott, R., Abbott, T. D., et al. 2017a, ApJ, 848, L13 —. 2017b, Physical Review Letters, 119, 161101

Albert, A., André, M., Anghinolfi, M., et al. 2019, MNRAS, 482, 184 Alexander, K. D., Berger, E., Fong, W., et al. 2017, ApJ, 848, L21

Alexander, K. D., Margutti, R., Blanchard, P. K., et al. 2018, ApJ, 863, L18

Amati, L., Della Valle, M., Frontera, F., et al. 2007, A\&A, 463, 913

Atwood, W. B., Abdo, A. A., Ackermann, M., et al. 2009, ApJ, 697, 1071

Bannister, K. W., Deller, A. T., Phillips, C., et al. 2019, arXiv e-prints, arXiv:1906.11476

Barthelmy, S. D., Barbier, L. M., Cummings, J. R., et al. 2005, Space Sci. Rev., 120, 143

Boella, G., Butler, R. C., Perola, G. C., et al. 1997, A\&AS, 122, 299

Bucciantini, N., Quataert, E., Arons, J., Metzger, B. D., \& Thompson, T. A. 2007, MNRAS, 380, 1541

Caleb, M., Stappers, B. W., Rajwade, K., \& Flynn, C. 2019, MNRAS, 484, 5500

Campana, S., Mangano, V., Blustin, A. J., et al. 2006, Nature, 442, 1008

Cappellaro, E., Botticella, M. T., Pignata, G., et al. 2015, A\&A, 584, A62

Chatterjee, S., Law, C. J., Wharton, R. S., et al. 2017, Nature, 541, 58

CHIME/FRB Collaboration, Amiri, M., Bandura, K., et al. 2019, Nature, 566,235

Cordes, J. M., \& Wasserman, I. 2016, MNRAS, 457, 232

Coward, D. M., Howell, E. J., Piran, T., et al. 2012, MNRAS, 425, 2668

Crawford, F., Rane, A., Tran, L., et al. 2016, MNRAS, 460, 3370

Dahlen, T., Strolger, L.-G., Riess, A. G., et al. 2004, ApJ, 613, 189

DeLaunay, J. J., Fox, D. B., Murase, K., et al. 2016, ApJ, 832, L1

Eftekhari, T., \& Berger, E. 2017, ApJ, 849, 162

Fan, Y.-Z., \& Xu, D. 2006, MNRAS, 372, L19

Feroci, M., Hurley, K., Duncan, R. C., \& Thompson, C. 2001, ApJ, 549, 1021

Fong, W., Berger, E., Margutti, R., \& Zauderer, B. A. 2015, ApJ, 815, 102

Frontera, F., Costa, E., dal Fiume, D., et al. 1997, A\&AS, 122, 357

Frontera, F., Guidorzi, C., Montanari, E., et al. 2009, ApJS, 180, 192

Gao, H., \& Zhang, B. 2017, ApJ, 835, L21

Gehrels, N., Chincarini, G., Giommi, P., et al. 2004, ApJ, 611, 1005

Gompertz, B. P., O’Brien, P. T., Wynn, G. A., \& Rowlinson, A. 2013, MNRAS, 431, 1745

Gourdji, K., Michilli, D., Spitler, L. G., et al. 2019, ApJ, 877, L19

Granot, J., Guetta, D., \& Gill, R. 2017, ApJ, 850, L24

Guidorzi, C., Lacapra, M., Frontera, F., et al. 2011, A\&A, 526, A49

H.E.S.S. Collaboration, Abdalla, H., Abramowski, A., et al. 2017, A\&A 597, A115

Howell, E. J., \& Coward, D. M. 2013, MNRAS, 428, 167

Hurley, K., Boggs, S. E., Smith, D. M., et al. 2005, Nature, 434, 1098

Jager, R., Mels, W. A., Brinkman, A. C., et al. 1997, A\&AS, 125, 557

Katz, J. I. 2014, Phys. Rev. D, 89, 103009

-. 2016, ApJ, 818, 19

- 2018, Progress in Particle and Nuclear Physics, 103, 1

Kisaka, S., Ioka, K., \& Sakamoto, T. 2017, ApJ, 846, 142

Kocevski, D., Ryde, F., \& Liang, E. 2003, ApJ, 596, 389

Kulkarni, S. R., Frail, D. A., Wieringa, M. H., et al. 1998, Nature, 395, 663

Kumar, P., \& Zhang, B. 2015, Phys. Rep., 561, 1

Lazzati, D., Ramirez-Ruiz, E., \& Ghisellini, G. 2001, A\&A, 379, L39

Li, W., Chornock, R., Leaman, J., et al. 2011, MNRAS, 412, 1473

Liang, E., Zhang, B., Virgili, F., \& Dai, Z. G. 2007, ApJ, 662, 111

Lorimer, D. R., Bailes, M., McLaughlin, M. A., Narkevic, D. J., \& Crawford, F. 2007, Science, 318, 777

Lyutikov, M., Burzawa, L., \& Popov, S. B. 2016, MNRAS, 462, 941

Macquart, J.-P., \& Ekers, R. D. 2018, MNRAS, 474, 1900

MAGIC Collaboration, Acciari, V. A., Ansoldi, S., et al. 2018, MNRAS, 481,2479
Margutti, R., Berger, E., Fong, W., et al. 2017, ApJ, 848, L20

Margutti, R., Alexander, K. D., Xie, X., et al. 2018, ApJ, 856, L18

Mazets, E. P., Golentskii, S. V., Ilinskii, V. N., Aptekar, R. L., \& Guryan, I. A. 1979, Nature, 282, 587

Meegan, C., Lichti, G., Bhat, P. N., et al. 2009, ApJ, 702, 791

Metzger, B. D., Berger, E., \& Margalit, B. 2017, ApJ, 841, 14

Metzger, B. D., Giannios, D., Thompson, T. A., Bucciantini, N., \& Quataert, E. 2011, MNRAS, 413, 2031

Metzger, B. D., Quataert, E., \& Thompson, T. A. 2008, MNRAS, 385, 1455

Montanari, E., Frontera, F., Guidorzi, C., \& Rapisarda, M. 2005, ApJ, 625, L17

Nakar, E., \& Sari, R. 2012, ApJ, 747, 88

Nicholl, M., Williams, P. K. G., Berger, E., et al. 2017, ApJ, 843, 84

Niino, Y. 2018, ApJ, 858, 4

Norris, J. P., \& Bonnell, J. T. 2006, ApJ, 643, 266

Palaniswamy, D., Li, Y., \& Zhang, B. 2018, ApJ, 854, L12

Palaniswamy, D., Wayth, R. B., Trott, C. M., et al. 2014, ApJ, 790, 63

Palmer, D. M., Barthelmy, S., Gehrels, N., et al. 2005, Nature, 434, 1107

Petroff, E., Hessels, J. W. T., \& Lorimer, D. R. 2019a, The Astronomy and Astrophysics Review, 27, 4

Petroff, E., Barr, E. D., Jameson, A., et al. 2016, PASA, 33, e045

Petroff, E., Oostrum, L. C., Stappers, B. W., et al. 2019b, MNRAS, 482, 3109

Planck Collaboration, Ade, P. A. R., Aghanim, N., et al. 2016, A\&A, 594, A13

Popov, S. B., Postnov, K. A., \& Pshirkov, M. S. 2018, Physics-Uspekhi, 61, 965

Rane, A., \& Lorimer, D. 2017, Journal of Astrophysics and Astronomy, 38, 55

Rane, A., Lorimer, D. R., Bates, S. D., et al. 2016, MNRAS, 455, 2207

Ravi, V. 2019, MNRAS, 482, 1966

Ravi, V., Catha, M., D’Addario, L., et al. 2019, arXiv e-prints, arXiv: 1907.01542

Romero, G. E., del Valle, M. V., \& Vieyro, F. L. 2016, Phys. Rev. D, 93, 023001

Rowlinson, A., O'Brien, P. T., Tanvir, N. R., et al. 2010, MNRAS, 409, 531

Scholz, P., Spitler, L. G., Hessels, J. W. T., et al. 2016, ApJ, 833, 177

Scholz, P., Bogdanov, S., Hessels, J. W. T., et al. 2017, ApJ, 846, 80

Shannon, R. M., \& Ravi, V. 2017, ApJ, 837, L22

Shannon, R. M., Macquart, J. P., Bannister, K. W., et al. 2018, Nature, 562, 386

Soderberg, A. M., Kulkarni, S. R., Nakar, E., et al. 2006, Nature, 442, 1014 Spitler, L. G., Scholz, P., Hessels, J. W. T., et al. 2016, Nature, 531, 202

Tendulkar, S. P., Kaspi, V. M., \& Patel, C. 2016, ApJ, 827, 59

Tendulkar, S. P., Bassa, C. G., Cordes, J. M., et al. 2017, ApJ, 834, L7

Thompson, C. 1994, MNRAS, 270, 480

Thornton, D., Stappers, B., Bailes, M., et al. 2013, Science, 341, 53

Troja, E., Lipunov, V. M., Mundell, C. G., et al. 2017, Nature, 547, 425

Tsvetkova, A., Frederiks, D., Golenetskii, S., et al. 2017, ApJ, 850, 161

Usov, V. V. 1992, Nature, 357, 472

Virgili, F. J., Liang, E.-W., \& Zhang, B. 2009, MNRAS, 392, 91

Wanderman, D., \& Piran, T. 2010, MNRAS, 406, 1944

-. 2015, MNRAS, 448, 3026

Waxman, E., Mészáros, P., \& Campana, S. 2007, ApJ, 667, 351

Xi, S.-Q., Tam, P.-H. T., Peng, F.-K., \& Wang, X.-Y. 2017, ApJ, 842, L8

Xu, J., \& Han, J. L. 2015, Research in Astronomy and Astrophysics, 15, 1629

Yamasaki, S., Totani, T., \& Kawanaka, N. 2016, MNRAS, 460, 2875

Yang, Y.-H., Zhang, B.-B., \& Zhang, B. 2019, ApJ, 875, L19

Zhang, B. 2018, ApJ, 867, L21

Zhang, B.-B., \& Zhang, B. 2017, ApJ, 843, L13

Zhang, S.-B., Hobbs, G., Dai, S., et al. 2019, MNRAS, 484, L147

APPENDIX

\section{BACKGROUND MODELING}

We conveniently split the data set into individual orbits: the data available for each orbit consist of 1-s ratemeters continuously acquired in both 40-700 and $>100 \mathrm{keV}$ energy bands, covering the time interval from the end of a given passage over the South Atlantic Anomaly to the beginning of the next one. Hereafter, times are referred to the FRB time. The FRB orbit, spanning from 
-2200 to $+2770 \mathrm{~s}$, is affected by no classified transient event, such as GRBs (Frontera et al. 2009), solar X-ray flares, or magnetar outburts. We estimated the background rates for each unit and energy band using the data of the fifteenth previous orbit (hereafter, referred to as the "background orbit"), during which the spacecraft had the same attitude as for the FRB orbit. The central time of the background orbit precedes that of the FRB one by 85505 s, i.e. only 655 s difference from a sidereal day: this way, not only do both orbits experience the same configuration with respect to the Earth magnetic field, but they also share a very similar visible portion of the sky. Data were cleaned from a few spikes due to high-energy charged particles: this was done by replacing the $>7 \sigma$ excesses with respect to a moving average with statistical realizations of the local average, after verifying that the same excesses were not present in different detectors, so as to exclude an electromagnetic wave origin. We therefore interpolated the rates of the background orbit in terms of Legendre polynomials up to degree 20, upon renormalizing the time span of the orbit to the $[-1,1]$ interval. The reason behind this choice lies in their orthogonality and easier comparison between the variance of a given time series, as described by its Legendre spectrum, and that of another series, with respect to using generic polynomials. A more detailed justification will be supplied in a future dedicated work. The quality of the interpolation was verified by applying a normality test on the residual 6 , a $\chi^{2}$ test, and a runs tes 17 to ensure that no trend was present. All the corresponding p-values were above the $1 \%$ threshold.

We then subtracted the background model for each unit and energy band from the corresponding data of the FRB orbit and checked the quality of the result through the same statistics as above: the worst reduced $\chi^{2}$ was 1.12 , while the lowest $\mathrm{p}$-value for the runs test was $5 \%$, thus confirming the robustness of the background modeling. This procedure benefited from the low variability of the equatorial orbit of BeppoSAX (Frontera et al. 1997).

${ }^{6}$ We used the python function "normaltest", based on D'Agostino and Pearson's test, as implemented in the SCIPY.STATS package.
7 We used the python function "wald_wolfowitz" from the SKIDMARKS package. 